\title{
Remote Sensing Image Fusion using Contourlet Transform With SHarp Frequency Localization
}

\author{
Yoonsuk Choi*, Ershad Sharifahmadian, Shahram Latifi \\ Dept. of Electrical and Computer Engineering, University of Nevada, Las Vegas \\ 4505 Maryland Parkway, Las Vegas, NV 89154-4026
}

\begin{abstract}
This paper addresses four different aspects of the remote sensing image fusion: i) image fusion method, ii) quality analysis of fusion results, iii) effects of image decomposition level, and iv) importance of image registration. First, a new contourlet-based image fusion method is presented, which is an improvement over the wavelet-based fusion. This fusion method is then utilized withinthe main fusion process to analyze the final fusion results. Fusion framework, scheme and datasets used in the study are discussed in detail. Second, quality analysis of the fusion results is discussed using various quantitative metrics for both spatial and spectral analyses. Our results indicate that the proposed contourlet-based fusion method performs better than the conventional wavelet-based fusion methodsin terms of both spatial and spectral analyses. Third, we conducted an analysis on the effects of the image decomposition level and observed that the decomposition level of 3 produced better fusion results than both smaller and greater number of levels. Last, we created four different fusion scenarios to examine the importance of the image registration. As a result, the feature-based image registration using the edge features of the source images produced better fusion results than the intensity-based imageregistration.
\end{abstract}

\section{KEYWORDS}

Contourlet, Hyperspectral, Multispectral, Panchromatic, Wavelet

\section{INTRODUCTION}

Remote sensing technologies provide remotely observed images with high spatial and spectral resolutions. However, due to the hardware limitations, current remote sensors provide spectral images that have low spatial resolution; hence, image fusion becomes necessary in order to fuse spectral images with high-resolution spatial images. The resultant fusion image provides scientists with more useful information both spatially and spectrally [1].

Recently, a new transform technique namely wavelet transform (WT) has been widely used in the field of image fusion due to its multi-resolution decomposition where input images are decomposed into different scales or levels of frequencies. However, the wavelet-based fusion techniques are good in detecting discontinuities at edge points but not optimal in capturing twodimensional singularities from the input images. Moreover, thesewavelets exhibit limited capabilities in detecting the smoothness along the contours of the input images [2]. Do and Vetterli introduced a new two-dimensional contourlet transform (CT) [3]. This transform is capable of constructing both multi-resolution and multi-directional expansions using a nonseparable pyramid directional filter bank (PDFB) [4]. 
Despite the advantages, Do's group has enhanced the original contourlet transform theory by developingnew contourlet-based transforms in order to overcome some drawbacks of the original contourlet transform. The first one is the nonsubsampled contourlet transform (NSCT) [5] and the second one is the contourlet transform with sharp frequency localization (CTSFL) [6].

In this paper, we mainly focus on the contourlet transform with sharp frequency localization due to its advantages in image processing, and present a new remote sensing image fusion method based on CTSFL. In Section 2 and Section 3, we discuss the principle, advantages and drawbacks of CT and CTSFL respectively. In Section 4, we present a new image fusion method based on CTSFL including the fusion framework and fusion scheme in detail. Then, we discuss the experiments and analyze the fusion results in Section 5, and conclusion is provided in Section 6.

\section{CONTOUR Let Transform}

The contourlet transform is a multi-scale and multi-direction framework of discrete image. The multi-scale and multi-directional analyses are separated in series. First, a Laplacian pyramid (LP) [7] is used to capture point discontinuities in input images.Second, a directional filter bank (DFB) [8] is applied to form linear structures by linkingthe point discontinuities. As a result,it becomespossible to obtain an image expansion using basic elements like contour segments. Thecontourlet transform framework is shown in Figure 1.

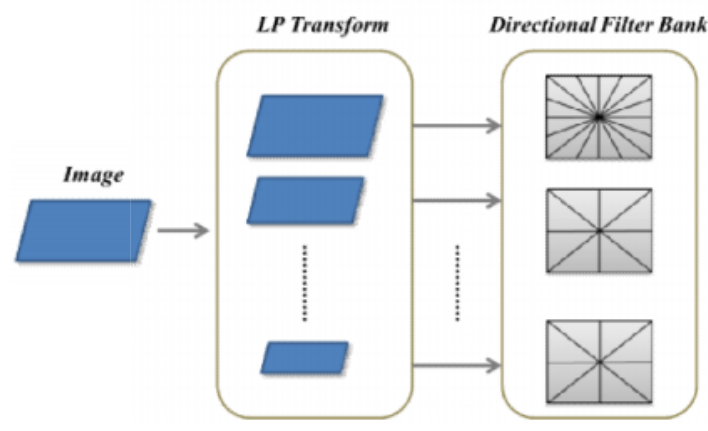

Figure 1. The contourlet transform framework

Figure 2 shows the contourlet filter bank. First, multi-scale decomposition is performed by the Laplacian pyramid, and then a directional filter bank is applied to each band pass channel.

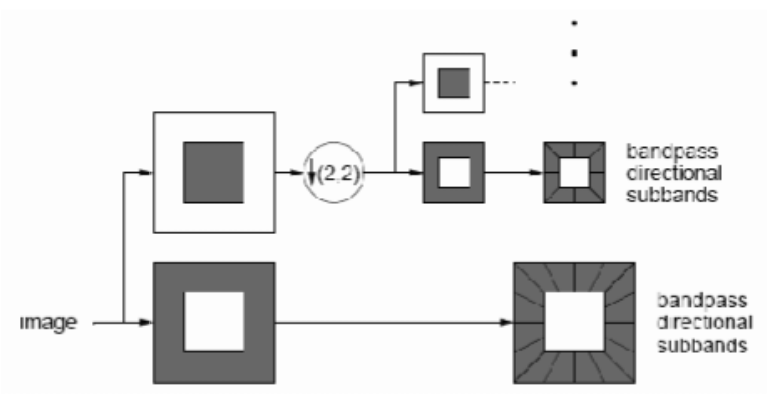

Figure 2. The contourlet filter bank

The contourlet expansion of images consists of basis images oriented at various directions in multiple scales with flexible aspect ratio. In addition to retaining the multi-scale and time- 
frequency localization properties of wavelets, the contourlet transform offers high degree of directionality. The contourlet transform adopts nonseparable basis functions, which makes it capable of capturing the geometrical smoothness of the contour along any possible direction. Compared to the traditional image expansions, the contourlets can capture 2-D geometrical structure in natural images much more efficiently [9].

Figure 3 shows a few wavelet and contourlet basis images. We can observe that the contourlets offer a much richer set of directions and shapes; hence, they are more suitable in capturing smooth contours and geometric structures in images.
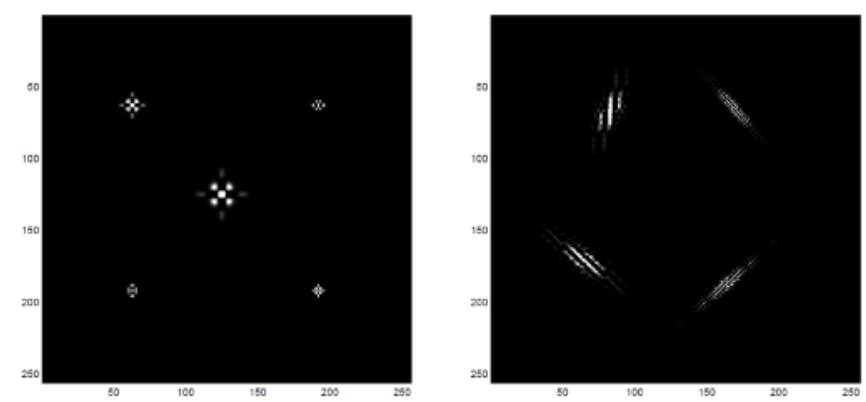

Figure 3. Comparison between actual 2-D wavelets (left) and contourlets (right) [3]

\section{CONTOURLET TransForm WiTh SHARP FrEQUENCY LOCALIZATION}

The major drawback of the original contourlet construction is that its basis images are not localized in the frequency domain. The contourlet transform is constructed as a combination of the Laplacian pyramid [10] and the directional filter banks (DFB) [8]. Conceptually, the flow of operation can be illustrated by Figure 4(a), where the Laplacian pyramid iteratively decomposes a 2-D image into lowpass and highpass sub-bands, and the DFB are applied to the highpass subbands to further decompose the frequency spectrum. Using ideal filters, the contourlet transform will decompose the 2-D frequency spectrum into trapezoid-shaped regions as shown in Figure 4(b).

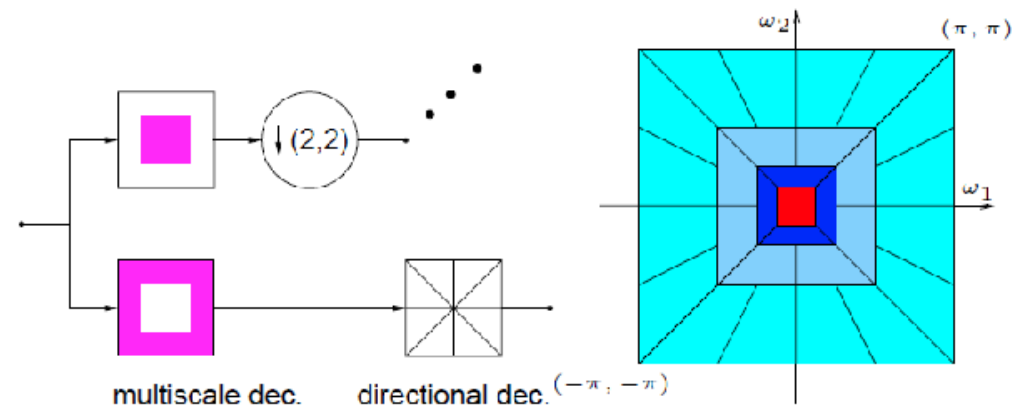

(a)

(b)

Figure 4.The original contourlet transform. (a) Block diagram. (b) Resulting frequency division

When non-ideal filters are used, the resulting contourlets do not have the desired sharp frequency domain localization. Although the majority of the energy in each subband isconcentrated on the ideal support regions, there are also significant amount of aliasing components at locations far away from the desired support. This type of frequency aliasing is undesirable because the 
resulting contourlets in the spatial domain are not smooth along their main ridges and exhibit some fuzzy artifacts. As a result, the efficiency is degraded in representing smooth boundaries in natural images [11]. This phenomenon has also beenobserved by other researches, such as the study by Candes et al. [12].

Y. Lu and M. N. Do proposed a new method of constructing the contourlet transform to overcome the drawback [11]. Figure 5 is a new construction of the contourlet transform. Directional filter bank is still used for directional decomposition; however, a significant difference is that a new pyramid structure for the multi-scale decomposition is used instead of using the Laplacian pyramid [13]. As a result, this new multi-scale pyramid can employ a different set of lowpass and highpass filters for the first level and all other levels [11].

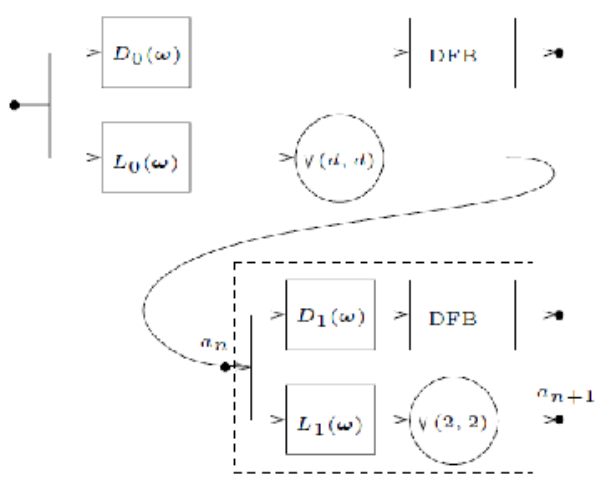

Figure 5.The new block diagram. Only the analysis part is shown, while the synthesis part is exactly symmetric [11]

\section{IMAGE FUSION}

\subsection{Fusion Framework}

The fusion framework used in our experiments is depicted in Figure 6. The general idea is identical to the image fusion using the original contourlet transform. The source images are decomposed using contourlet transform and the components are fused together according to a certain fusion scheme. Then, the final fusion result is obtained by performing inverse contourlet transform. However, the main difference is the multi-scale decomposition. In other words, the directional filter bank is still used for directional decomposition, but a new pyramid structure for the multi-scale decomposition is used instead of using the Laplacian pyramid. This new multiscale pyramid can utilize a different set of lowpass and highpass filters for the first level and all other levels.

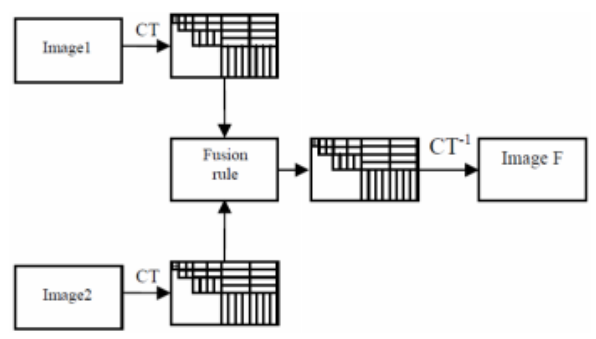

Figure 6.The fusion framework based on CTSFL 


\subsection{Fusion Scheme}

The source images are first decomposed into multi-scale and multi-directional images using CTSFL, and then these decomposed images are fused based on a certain fusion scheme. Since the low frequency parts include mostly the background information, we need weighted-average operators. In other words, the low frequency coefficients are weight-averaged. On the other hand, the high frequency parts include mostly the image representation information, such as edge and texture information. The correlation between a pixel and its neighboring pixels is often larger than others. Hence, the fusion scheme should know about the region where the pixel is the center. The fusion scheme computes the region energy of the center pixel and its neighboring pixels. The expression of region energy is as follows:

$$
\begin{aligned}
& E_{A}(i, j)=\sum_{(m, n) \in w} \omega(m, n) f_{A}^{2}(m, n) \\
& E_{B}(i, j)=\sum_{(m, n) \in w} \omega(m, n) f_{B}^{2}(m, n)
\end{aligned}
$$

, where $i-k \leq m \leq i+k, j-k \leq n \leq j+k$, width of the region is $w(w=2 k+1), \omega(m, n)$ is the weighted value and $f(m, n)$ is the pixel gray value.

The region energy is larger when the image features are salient; hence, the region energy for each pixel is compared and high frequency coefficients of the pixel with larger region energy are selected to be used as high frequency coefficients of the fused image. This way, the salient image features, such as edge and texture information can be preserved [14, 15].

Based on the above discussion, the region-based measure fusion operators are used in the experiments. First, the region is selected using a 3-by-3 window. Second, if the difference of the region energy of pixels from the source images is large, select high frequency coefficients of the pixel with larger region energy. Otherwise, perform weighted-average over the pixels' high frequency coefficients as follows:

$$
F(i, j)=\left\{\begin{array}{cc}
A(i, j), & E_{A(i, j)}>E_{B(i, j)}, R_{A B}(i, j)>T \\
B(i, j), & E_{A(i, j)}<E_{B(i, j)}, R_{A B}(i, j)>T \\
\omega_{1} A(i, j)+\omega_{2} B(i, j), & R_{A B}(i, j) \leq T
\end{array}\right.
$$

where $T$ is the energy match degree threshold and $\omega_{1}+\omega_{2}=1$. The energy matching degree $R_{A B}(i, j)$ as follows:

$$
R_{A B}(i, j)=\frac{2 \sum_{(m, n) \in w} \omega(m, n) f_{A}(m, n) f_{B}(m, n)}{E_{A}(i, j)+E_{B}(i, j)}
$$


where $R_{A B}(i, j)$ value is between 0 and 1 .

According to this fusion scheme, the high frequency coefficients of the salient feature region can be preserved. Lastly, the inverse transform is performed to reconstruct the fused image [15].

\section{EXPERIMENTAL STUDY AND ANALYSIS}

In our experiments, three different datasets are used: i) a pair of multispectral (MS) and panchromatic (PAN) images, and ii) two pairs of hyperspectral (HS) and panchromatic (PAN) images. Each of the dataset is explained more in detail in subsections 5.1 and 5.2 respectively. Moreover, the following sub-sections explain how the pre-processing is performed over the source images prior to the image fusion.

In order to quantitatively analyze the fusion results, we employ various quality metrics, and they can be classified into two categories: i) spectral analysis and ii) spatial analysis. Correlation coefficient (CC) [16], relative average spectral error (RASE) [17], spectral angle mapper (SAM) [18] and spectral information divergence (SID) [19] are used for spectral analysis. On the other hand, for spatial analysis, we employ entropy (E) [20, 21], universal image quality index (UIQI) [22], signal-to-noise ratio (SNR) and average gradient (AG) [23].

\subsection{Multispectral and Panchromatic Images (Dataset 1)}

The first dataset was downloaded from [24]. This is a set of 4m-MS and 1m-PAN images of the city of Fredericton, Canada which were acquired by the commercial satellite IKONOS. PAN image is resampled to match the size of MS image, and it is assumed that they are co-registered to each other. Since we do not have a reference image to be compared to the fusion results, we spatially degraded the original MS to obtain a source image. In other words, we use the degraded MS image as a source image and the original MS image as a reference image. The fusion results are quantitatively compared to the original MS image to perform both spectral and spatial analyses.

Two source images (PAN and MS) and the reference MS image are shown in Figure 7.

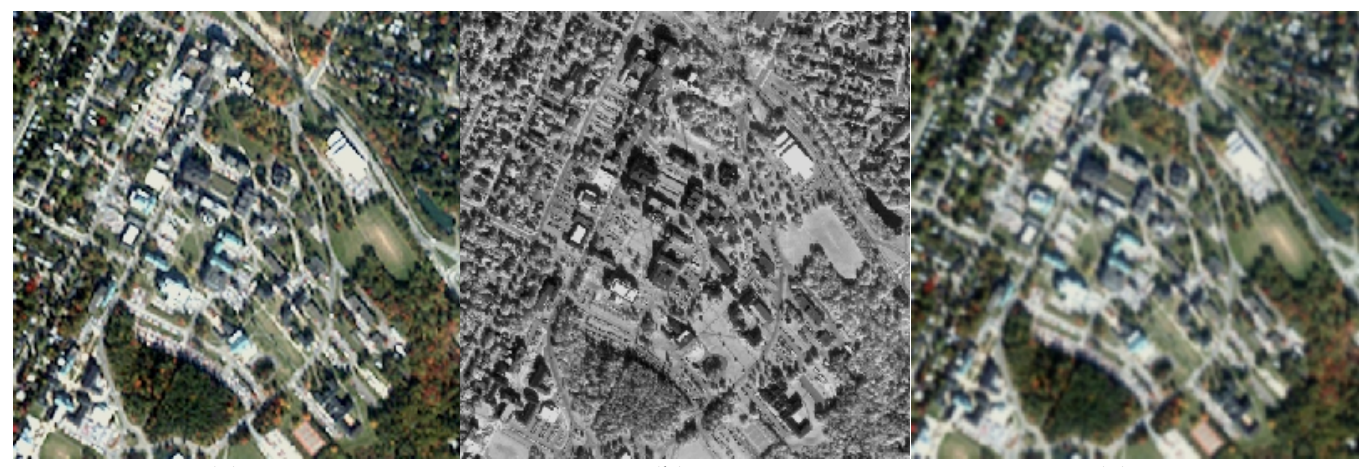

(a)

(b)

(c)

Figure 7.Dataset 1 - the original MS image and two synthesized source images. (a) Original (reference) MS image. (b) Synthesized PAN source image. (c) Synthesized MS source image

Fusion of the above source images is performed based on CTSFL and fusion scheme as discussed in the previous section. The fused result is analyzed using various quality metrics as discussed 
earlier. In order to validate and analyze our fusion method, we compared the proposed fusion method to two well-known wavelet-based fusion methods: i) substitutive wavelet transform fusion (SWT) and ii) additive wavelet transform scheme (AWT). The fusion results are shown in Figure 8, and performance comparison of the results is shown in Table 1.

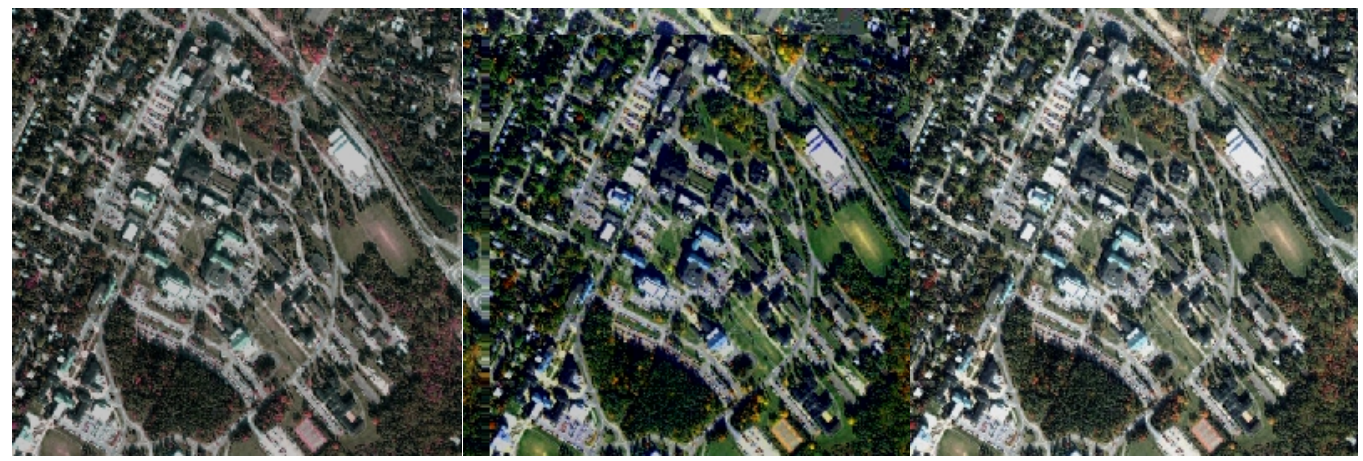

(a)

(b)

(c)

Figure 8.Fusion results of dataset 1. (a) SWT. (b) AWT. (c) CTSFL

Table 1.A performance comparison of the fusion results using quality assessment metrics for Dataset 1.

\begin{tabular}{|c|c|c|c|c|c|c|c|c|}
\hline \multirow{2}{*}{$\begin{array}{c}\text { Fusion } \\
\text { Method }\end{array}$} & \multicolumn{4}{|c|}{ Spectral Analysis } & \multicolumn{4}{c|}{ Spatial Analysis } \\
\cline { 2 - 9 } & $\boldsymbol{C C}$ & $\boldsymbol{R A S E}$ & $\boldsymbol{S A M}$ & $\boldsymbol{S I D}$ & $\boldsymbol{E}$ & $\boldsymbol{U} \boldsymbol{I} \boldsymbol{I}$ & $\boldsymbol{S N R}$ & $\boldsymbol{A G}$ \\
\hline \hline SWT & 0.843 & 45.756 & 0.238 & 0.222 & 5.769 & 0.771 & 68.724 & 4.689 \\
\hline AWT & $\mathbf{0 . 8 7 6}$ & $\mathbf{4 3 . 7 1 1}$ & 0.227 & 0.217 & 6.235 & 0.797 & 69.419 & 5.617 \\
\hline CTSFL & 0.864 & 43.735 & $\mathbf{0 . 2 1 6}$ & $\mathbf{0 . 1 6 4}$ & $\mathbf{6 . 4 2 5}$ & $\mathbf{0 . 8 2 3}$ & $\mathbf{7 1 . 4 6 3}$ & $\mathbf{6 . 1 2 6}$ \\
\hline
\end{tabular}

As we can see from Table 1, our proposed method is validated using eight different quality metrics. CTSFL method performs better than the other two wavelet-based methods in terms of spatial analysis; however, AWT method performs better than CTSFL in terms of CC and RASE. This may have happened due to the pre-processing step that we went through. If co-registration is properly done, the spectral analysis results may have been different. However, there are various image registration methods, and thus each method may give different spectral and spatial results. The effect of pre-processing in image fusion needs to be further studied.

\subsection{Hyperspectral and Panchromatic Images (Dataset 2 and 3)}

The second and third datasets are hyperspectral images from MultiSpec@ $\odot$ homepage by Purdue University (MultiSpec(C) [25]. In this second experiment, we only have two original hyperspectral images; hence, we need to go through some pre-processing to obtain source images. First, the original hyperspectral image is down sampled by a factor of 3 and resized to the same size as before using the bilinear interpolation. The final result is a synthesized hyperspectral image that can be used in our experiment as a source image. Second, the original hyperspectral image is spectrally integrated over the entire spectral range to obtain a panchromatic image with spatial details preserved as much as possible. The final result is a synthesized panchromatic image that can be used as the second source image [26]. By doing this, we can obtain two perfectly coregistered source images without going through registration process which takes up lots of time and computational load. Figure 9 and 11 show the original HS image and two synthesized source images. Figure 10 and 12 show the fusion results of (a) SWT, (b) AWT and (c) CTSFL respectively. 


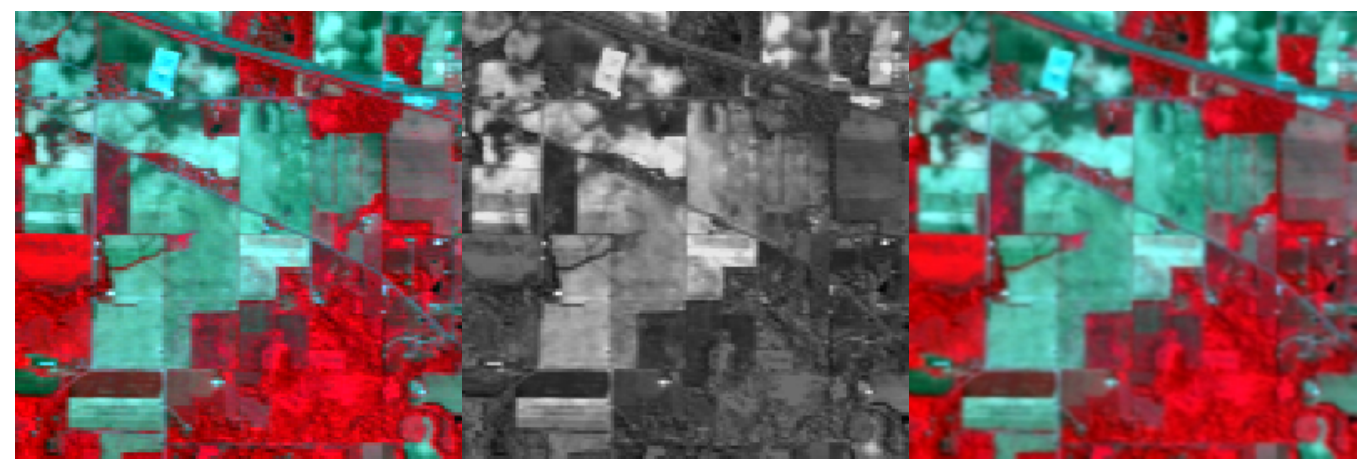

(a)

(b)

(c)

Figure 9.Dataset 2 - the original HS image and two synthesized source images. (a) Original (reference) HS image. (b) Synthesized PAN source image. (c) Synthesized HS source image

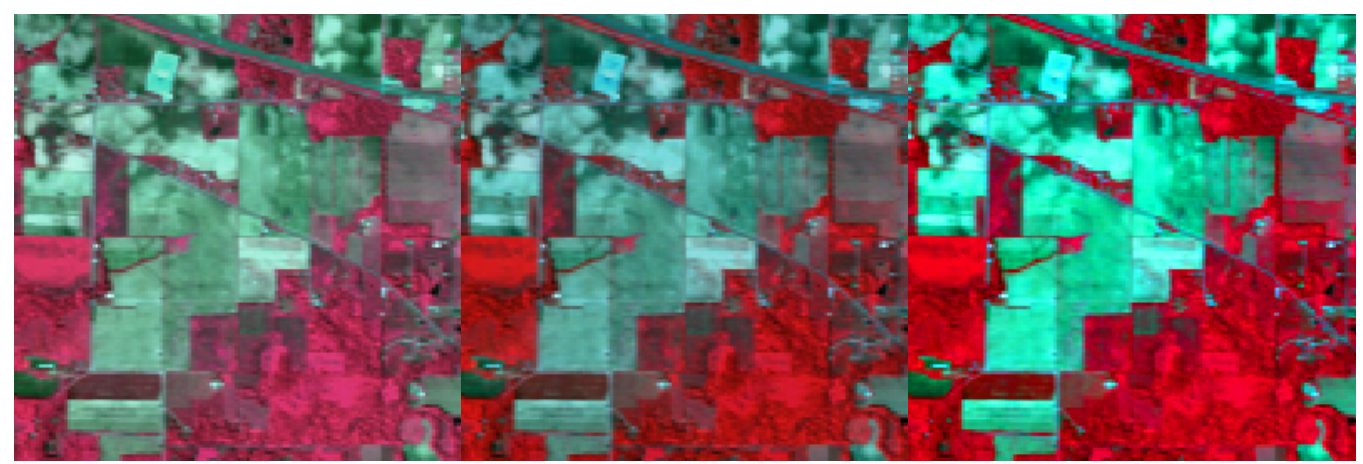

(a)

(b)

(c)

Figure 10.Fusion results of dataset 2. (a) SWT. (b) AWT. (c) CTSFL

Table 2.A performance comparison of the fusion results using quality assessment metrics for Dataset 2.

\begin{tabular}{|c|c|c|c|c|c|c|c|c|}
\hline \multirow{2}{*}{$\begin{array}{c}\text { Fusion } \\
\text { Method }\end{array}$} & \multicolumn{4}{|c|}{ Spectral Analysis } & \multicolumn{4}{c|}{ Spatial Analysis } \\
\cline { 2 - 9 } & $\boldsymbol{C C}$ & $\boldsymbol{R A S E}$ & $\boldsymbol{S A M}$ & $\boldsymbol{S I D}$ & $\boldsymbol{E}$ & $\boldsymbol{U} \boldsymbol{Q} \boldsymbol{I}$ & $\boldsymbol{S N R}$ & $\boldsymbol{A} \boldsymbol{G}$ \\
\hline \hline SWT & 0.821 & 43.834 & 0.257 & 0.187 & 6.593 & 0.774 & 68.654 & 5.383 \\
\hline AWT & 0.849 & 43.682 & 0.225 & 0.168 & 6.752 & 0.782 & 69.136 & 5.816 \\
\hline CTSFL & $\mathbf{0 . 8 7 5}$ & $\mathbf{4 3 . 4 2 6}$ & $\mathbf{0 . 2 0 2}$ & $\mathbf{0 . 1 4 3}$ & $\mathbf{6 . 8 5 1}$ & $\mathbf{0 . 8 4 3}$ & $\mathbf{7 2 . 7 7 3}$ & $\mathbf{6 . 7 3 2}$ \\
\hline
\end{tabular}


International Journal of Information Technology, Modeling and Computing (IJITMC) Vol. 2, No. 1, February 2014

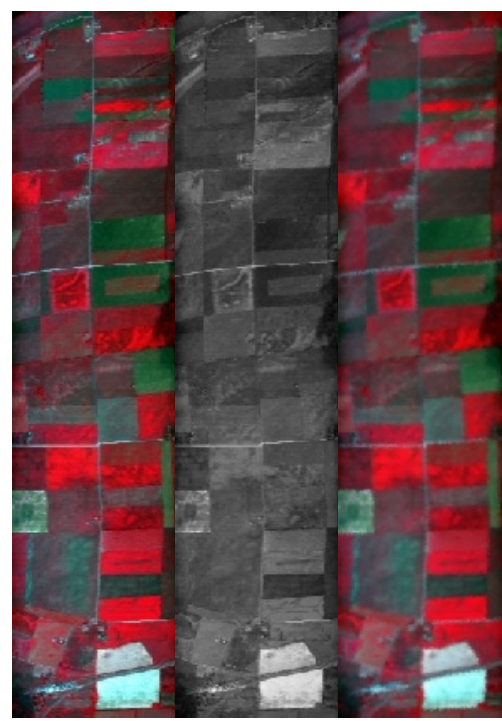

(a)

(b)

(c)

Figure 11.Dataset 3 - the original HS image and two synthesized source images. (a) Original (reference) HS image. (b) Synthesized PAN source image. (c) Synthesized HS source image

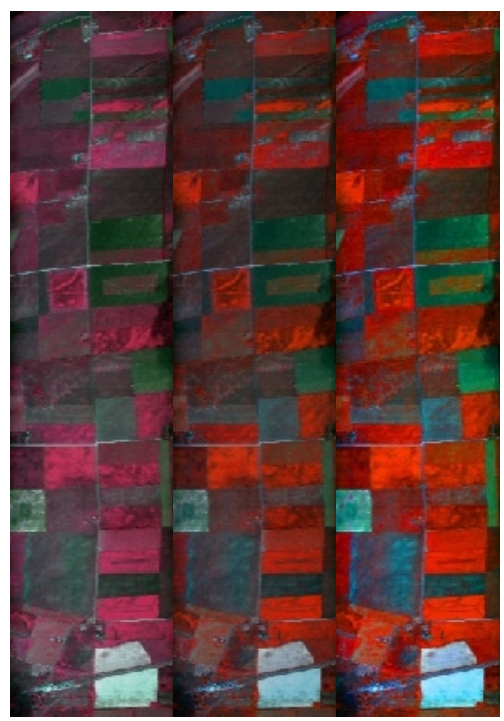

(a)

(b)

(c)

Figure 12.Fusion results of dataset 3. (a) SWT. (b) AWT. (c) CTSFL 
International Journal of Information Technology, Modeling and Computing (IJTMC) Vol. 2, No. 1, February 2014

Table 3.A performance comparison of the fusion results using quality assessment metrics for Dataset 3.

\begin{tabular}{|c|c|c|c|c|c|c|c|c|}
\hline \multirow{2}{*}{$\begin{array}{c}\text { Fusion } \\
\text { Method }\end{array}$} & \multicolumn{4}{|c|}{ Spectral Analysis } & \multicolumn{4}{c|}{ Spatial Analysis } \\
\cline { 2 - 9 } & $\boldsymbol{C C}$ & $\boldsymbol{R A S E}$ & $\boldsymbol{S A M}$ & $\boldsymbol{S I D}$ & $\boldsymbol{E}$ & $\boldsymbol{U I Q I}$ & $\boldsymbol{S N R}$ & $\boldsymbol{A G}$ \\
\hline \hline SWT & 0.792 & 43.942 & 0.251 & 0.198 & 6.383 & 0.754 & 67.459 & 5.374 \\
\hline AWT & 0.828 & 43.783 & 0.238 & 0.184 & 6.542 & 0.768 & 68.362 & 5.726 \\
\hline CTSFL & $\mathbf{0 . 8 7 5}$ & $\mathbf{4 3 . 7 1 1}$ & $\mathbf{0 . 2 1 2}$ & $\mathbf{0 . 1 5 7}$ & $\mathbf{6 . 7 3 1}$ & $\mathbf{0 . 8 3 2}$ & $\mathbf{7 1 . 8 3 7}$ & $\mathbf{6 . 3 7 5}$ \\
\hline
\end{tabular}

Similar to the first experiment with dataset 1, the experiments with dataset 2 and 3 show that our proposed method performs well and its effectiveness as a fusion method is validated. However, there is a notable difference between the results of dataset 1 and dataset $2 \& 3$. In other words, unlike dataset 1, CTSFL outperforms SWT and AWT both spectrally and spatially based on eight different quality metrics when using dataset 2 and 3. With the dataset 1 , AWT seems to be performing better than CTSFL in terms of CC and RASE; however, this may have happened because of the way the source images were pre-processed. Dataset 2 and 3 were pre-processed to obtain two perfectly co-registered source images; on the other hand, dataset 1 was pre-processed differently assuming that the original source images were co-registered to each other.

Table 4.A close comparison of the fusion results of CTSFL.

\begin{tabular}{|c|c|c|c|c|c|c|c|c|}
\hline \multirow{2}{*}{ CTSFL } & \multicolumn{4}{|c|}{ Spectral Analysis } & \multicolumn{4}{c|}{ Spatial Analysis } \\
\cline { 2 - 9 } & $\boldsymbol{C C}$ & $\boldsymbol{R A S E}$ & $\boldsymbol{S A M}$ & $\boldsymbol{S I D}$ & $\boldsymbol{E}$ & $\boldsymbol{U I Q I}$ & $\boldsymbol{S N R}$ & $\boldsymbol{A G}$ \\
\hline \hline Dataset 1 & 0.864 & 43.735 & 0.216 & 0.164 & 6.425 & 0.823 & 71.463 & 6.126 \\
\hline Dataset 2 & $\mathbf{0 . 8 8 6}$ & $\mathbf{4 3 . 4 2 6}$ & $\mathbf{0 . 2 0 2}$ & $\mathbf{0 . 1 4 3}$ & $\mathbf{6 . 8 5 1}$ & $\mathbf{0 . 8 4 3}$ & $\mathbf{7 2 . 7 7 3}$ & $\mathbf{6 . 7 3 2}$ \\
\hline Dataset 3 & 0.875 & 43.711 & 0.212 & 0.157 & 6.731 & 0.832 & 71.837 & 6.375 \\
\hline
\end{tabular}

Table 4 summarizes the fusion results of CTSFL on dataset 1, 2 and 3. This table is presented to provide a comparison of the fusion results of CTSFL alone. As shown in the table, we can observe that the results on dataset 2 and 3 are better than the results on dataset 1, and the reason is because the pre-processing was done differently. In other words, unlike the dataset 1 , the original hyperspectral image was pre-processed to obtain two synthesized source images (panchromatic and hyperspectral) that were perfectly co-registered. Moreover, if we take a closer look at dataset 2 and 3 only, we can observe that dataset 2 shows better results than dataset 3 in terms of all the quality metrics. First, in spectral analysis, dataset 2 has higher correlation coefficient than dataset 3 because the original hyperspectral image in dataset 2 consists of 220 bands while the original hyperspectral image in dataset 3 includes only 12 bands. Therefore, dataset 2 showed better spectral results by preserving more spectral information with higher correlation between the bands. Second, in spatial analysis, the fusion results on dataset 2 are better than the results on dataset 3 . The reason is because in both datasets, the original hyperspectral image was spectrally integrated to obtain a high-resolution panchromatic image as a source image. However, due to a difference in number of the spectral bands of the original hyperspectral image, the spatial details were preserved well in dataset 2 . As a result, the spatial performance of the fusion results using dataset 2 was higher. 
Table 5.A comparison of the fusion results with different levels of decomposition.

\begin{tabular}{|c|c|c|c|c|c|c|c|c|}
\hline \multirow{2}{*}{$\begin{array}{c}\text { Decomposition } \\
\text { Level }\end{array}$} & \multicolumn{4}{|c|}{ Spectral Analysis } & \multicolumn{4}{c|}{ Spatial Analysis } \\
\cline { 2 - 9 } & $\boldsymbol{C C}$ & $\boldsymbol{R A S E}$ & $\boldsymbol{S A M}$ & $\boldsymbol{S I D}$ & $\boldsymbol{E}$ & $\boldsymbol{U I Q I}$ & $\boldsymbol{S N R}$ & $\boldsymbol{A G}$ \\
\hline \hline Level 1 & 0.746 & 47.357 & 0.256 & 0.234 & 6.225 & 0.632 & 69.463 & 5.926 \\
\hline Level 2 & 0.823 & 45.264 & 0.232 & 0.193 & 6.451 & 0.734 & 70.773 & 6.132 \\
\hline Level 3 & $\mathbf{0 . 8 7 5}$ & $\mathbf{4 3 . 7 1 1}$ & $\mathbf{0 . 2 1 2}$ & $\mathbf{0 . 1 5 7}$ & $\mathbf{6 . 7 3 1}$ & $\mathbf{0 . 8 3 2}$ & $\mathbf{7 1 . 8 3 7}$ & $\mathbf{6 . 3 7 5}$ \\
\hline Level 4 & 0.819 & 46.925 & 0.247 & 0.221 & 6.158 & 0.779 & 68.962 & 6.021 \\
\hline
\end{tabular}

Since it has been verified that the CTSFL outperforms other wavelet-based methods in fusing remote sensing images, we have analyzed one more aspect of the image fusion which is the decomposition level. Dataset 3 was used to analyze how the decomposition level affects the fusion results. As Table 5 shows, the decomposition level of 3 provided the best fusion result, compared to the levels that are smaller or greater than the level 3. Further studies are necessary on this topic of the decomposition level because the analysis results may vary based on which type or modality of dataset is used in the fusion.

Table 6.An analysis on the importance of the image registration.

\begin{tabular}{|c|c|c|c|c|c|c|c|c|}
\hline \multirow{2}{*}{$\begin{array}{c}\text { Image } \\
\text { Registration }\end{array}$} & \multicolumn{4}{|c|}{ Spectral Analysis } & \multicolumn{4}{c|}{ Spatial Analysis } \\
\cline { 2 - 9 } & $\boldsymbol{C C}$ & $\boldsymbol{R A S E}$ & $\boldsymbol{S A M}$ & $\boldsymbol{S I D}$ & $\boldsymbol{E}$ & $\boldsymbol{U I Q I}$ & $\boldsymbol{S N R}$ & $\boldsymbol{A} \boldsymbol{G}$ \\
\hline \hline Case 1 & 0.582 & 52.569 & 0.458 & 0.364 & 5.925 & 0.502 & 67.643 & 5.716 \\
\hline Case 2 & 0.695 & 47.465 & 0.364 & 0.293 & 6.451 & 0.634 & 70.336 & 6.032 \\
\hline Case 3 & 0.755 & 46.731 & 0.312 & 0.237 & 6.613 & 0.683 & 71.748 & 6.457 \\
\hline Case 4 & $\mathbf{0 . 8 3 4}$ & $\mathbf{4 3 . 9 2 5}$ & $\mathbf{0 . 2 0 6}$ & $\mathbf{0 . 1 4 3}$ & $\mathbf{6 . 8 5 8}$ & $\mathbf{0 . 8 7 7}$ & $\mathbf{7 4 . 6 9 2}$ & $\mathbf{7 . 0 2 1}$ \\
\hline
\end{tabular}

Another important comparative analysis was conducted on the registration of the source (input) images using Dataset 3. Most of the remote sensing image fusion methods that are being studied today mainly focus on the fusion methods and rules. In other words, they fail to notice how important the registration of the source images is. Therefore, as shown in Table 6, four different cases have been compared and analyzed. In Case 1, two purposely misaligned source images are fused together. The source images were shifted horizontally and no image registration was performed here in order to produce the worst scenario. In Case 2, the same set of source images was used; however, we applied intensity-based image registration to align the source images. Intensity-based registration aligns two images by comparing the intensity patterns in each image via correlation metrics. In Case 3, the same set of source images was used; however, the featurebased image registration was applied to align the source images. Feature-based registration aligns two images by finding correspondence between the image features. In our case, we utilized the edge features that were extracted from the source images. For a point on the first image, it is expected to find the best matching point on the second image according to the edge features. Case 4 was the same as the previous experiments where two perfectly co-registered source images were used. As we can see from Table 6, Case 4 produced the best fusion results since the source images were perfectly registered to each other, and Case 1 obviously produced the worst fusion results since no registration was performed to the source images. From the comparison between Case 2 and Case 3, we observed that Case 3 produced better fusion results because the featurebased image registration performed better in aligning the source images by extracting the edge information. 


\section{CONClusions}

In this paper, we proposed a new fusion method based on contourlet transform with sharp frequency localization. Recently, contourlet transform has been utilized by many researchers in the field of image fusion due to its advantages over conventional wavelet-based methods. However, the contourlet transform has a major drawback, which is that its basis images are not localized in the frequency domain. A new method of constructing the contourlet transform to overcome the drawback is CTSFL, and this new idea was implemented in our fusion method. Fusion results of the proposed method were compared to well-known wavelet-based fusion methods, and the results were quantitatively analyzed using various quality metrics both spatially and spectrally. The performance comparison results show that our method outperforms the other two wavelet-based methods both spatially and spectrally; hence, the effectiveness of our fusion method is validated. Moreover, the effects of the decomposition level were tested and analyzed using Dataset 3. The comparison results show that the decomposition level of 3 provides better fusion results in terms of remote sensing image fusion. However, it is necessary to study this topic more in detail with various modalities because the fusion results may vary according to the modality of the dataset. It was also observed that the registration of the source images played an important role in the fusion process. Based on our experimental analysis, the feature-based image registration produced better fusion results than the intensity-based image registration. The effect and dependency of the source image pre-processing need to be further studied, and this area may be a good future research in remote sensing image fusion.

\section{ACKNOWLEDGEMENTS}

This work was supported in part by the Nevada EPSCoR program NSF award \#EPS-IIA-1301726 and by the DOD-DTRAGrant/Award \#: HDTRA1-12-1-0033.

\section{REFERENCES}

[1] G. Pajares and M. de la Cruz, "A wavelet-based image fusion tutorial”, Pattern Recognition, vol. 37 , no. 9, pp. 1855-1872, 2004.

[2] G. V. Welland, "Beyond Wavelets", Academic Press, 2003.

[3] M. N. Do and M. Vetterli, "The contourlet transform: An efficient directional multiresolution image representation”, IEEE Transactions on Image Processing, vol. 14, no. 12, pp. 2091-2106, 2005.

[4] S. Ibrahim and M. Wirth, "Visible and IR Data Fusion Technique Using the Contourlet Transform", International conference on computational science and engineering, CSE 09, IEEE, vol. 2, pp. 42-47, 2009.

[5] Da Cunha A L, Zhou J , Do M N, “The nonsubsampled contourlet transform: theory , design , and applications", IEEE Trans . Image Proc. ,15 (10) : 308923101, 2006.

[6] Yue Lu; Do, M.N., "A New Contourlet Transform with Sharp Frequency Localization", Image Processing, 2006 IEEE International Conference on, vol., no., pp.1629,1632, 8-11 Oct. 2006.

[7] Burt P J., "Merging images through pattern decomposition", Proceedings of SPIE, 575: 173-18, 1985.

[8] Bamberger R H., "A filter bank for the directional decomposition of images: Theory and design", IEEE Trans. Signal Processing, 40 (4): 882 -893, 1992.

[9] Aboubaker M. ALEjaily et al., "Fusion of remote sensing images using contourlet transform", Innovations and Advanced Techniques in Systems, Computing Sciences and Software Engineering, Springer, pp. 213-218, 2008.

[10] P. J. Burt and E. H. Adelson, "The Laplacian pyramid as a compact image code", IEEE Trans. Comm., vol. COM-31, pp. 532-540, April 1983.

[11] Yue Lu; Do, M.N., "A New Contourlet Transform with Sharp Frequency Localization," Image Processing, 2006 IEEE International Conference on , vol., no., pp.1629,1632, 8-11 Oct. 2006. 
[12] E. J. Cand'es, L. Demanet, D. L. Donoho, and L. Ying, "Fast discrete curvelet transforms", Tech. Rep., Applied and Computational Mathematics, California Institute of Technology, 2005.

[13] E. P. Simoncelli,W. T. Freeman, E. H. Adelson, and D. J. Heeger, "Shiftablemultiscale transforms," IEEE Trans. Inform. Th., Special Issue on Wavelet Transforms and Multiresolution Signal Analysis, vol. 38, no. 2, pp. 587-607, March 1992.

[14] P. J. Burt and R. J. Koiczynski, "Enhancement with application to image fusion", Proceeding of the 4th International Conference on Computer Vision, pp. 173-182, 1993.

[15] Lei Tang; Zong-Gui Zhao, "Multiresolution image fusion based on the wavelet-based contourlet transform”, Information Fusion, 2007 10th International Conference on , vol., no., pp.1,6, 9-12 July 2007.

[16] V. Vijayaraj, C.G.O. Hara, N.H. Younan, "Quality analysis of pansharpened images”, Geosci.Remote Sens. Symp. (2004) 85-88.

[17] T. Ranchin and L. Wald, "Fusion of high spatial and spectral resolution images: The ARSIS concept and its implementation," Photogramm. Eng. Remote Sens., vol. 66, no. 1, pp. 49-61, Jan. 2000.

[18] H.Z.M. Shafri, A. Suhaili, S. Manso, "The performance of Maximum likelihood spectral angle mapper neural network and decision tree classifiers in hyperspectral image analysis", J. Comput. Sci. 3 (6) (2007) 419-423.

[19] C.I. Chang, "An information theoretic-based approach to spectral variability, similarity and discriminability for hyperspectral image analysis", IEEE Trans. Inf. Theory 46 (5) (2000) 1927-1932.

[20] Y. Chen, Z. Xue, R.S. Blum, "Theoretical analysis of an information-based quality measure for image fusion", Inf. Fus. 2 (2008) 161-175.

[21] J.W. Roberts, J. Van Aardt, F. Ahmed, "Assessment of image fusion procedures using entropy, image quality, and multispectral classification”, J. Appl. Remote Sens. 1 (2008) 023522.

[22] Z.Wang and A. C. Bovik, “A universal image quality index”, IEEE Signal Process. Lett., vol. 9, no. 3, pp. 81-84, Mar. 2002.

[23] Li, Z., Jing, Z., Yang, X., Sun, S., "Color transfer based remote sensing image fusion using nonseparable wavelet frame transform”, Pattern Recognition Lett. 26 (13) (2005) 2006-2014.

[24] http://studio.gge.unb.ca/UNB/zoomview/examples.html

[25] MultiSpec@, https://engineering.purdue.edu/ biehl/MultiSpec/hyperspectral.html

[26] M. Eismann, R. Hardie, "Application of the stochastic mixing model to hyperspectral resolution enhancement”, IEEE Transactions on Geoscience and Remote Sensing 42 (9) (2004) 1924-1933. 